\title{
Deep brain stimulation telemedicine programming during the COVID-19 pandemic: treatment of patients with psychiatric disorders
}

\author{
Zhengyu Lin, MSc, ${ }^{1,2}$ Chencheng Zhang, MD, PhD, ${ }^{1-3}$ Yingying Zhang, MSc, ${ }^{1,2}$ Lulin Dai, MD, PhD, ${ }^{1,2}$ \\ Valerie Voon, MD, PhD, ${ }^{4}$ Dianyou Li, MD, PhD, ${ }^{1,2}$ and Bomin Sun, MD, PhD ${ }^{1,2}$ \\ ${ }^{1}$ Department of Neurosurgery and ${ }^{2}$ Center for Functional Neurosurgery, Ruijin Hospital, Shanghai Jiao Tong University School of \\ Medicine, Shanghai, China; ${ }^{3}$ Shanghai Research Center for Brain Science and Brain-Inspired Intelligence, Shanghai, China; and \\ ${ }^{4}$ Department of Psychiatry, University of Cambridge, Addenbrooke's Hospital, Cambridge, United Kingdom
}

\begin{abstract}
OBJECTIVE The ongoing coronavirus disease 2019 (COVID-19) pandemic has considerably affected the delivery of postoperative care to patients who have undergone deep brain stimulation (DBS) surgery. DBS teleprogramming technology was developed and deployed in China before the COVID-19 outbreak. In this report, the authors share their experiences with telemedical DBS treatment of patients with psychiatric disorders during the COVID-19 outbreak.

METHODS Four patients (2 with obsessive-compulsive disorder, 1 with major depressive disorder, and 1 with anorexia nervosa) underwent DBS surgery at Ruijin Hospital and received continuous postoperative DBS telemedicine case management from January 2020 to July 2020. DBS teleprogramming, individualized psychological support, and medical consultations were provided via the authors' DBS telemedicine platform, which also incorporated a synchronous realtime video communication system.

RESULTS Forty-five DBS telemedicine sessions were conducted; there was no unexpected loss of network connection during the sessions. Of these, 28 sessions involved DBS teleprogramming. Adjustments were made to the stimulation voltage, frequency, pulse width, and contact site in 21, 12, 9, and 9 sessions, respectively. Psychological support and troubleshooting were provided during the remaining telemedicine sessions. Modest to substantial clinical improvements after DBS surgery were observed in some but not all patients, whereas stimulation-related side effects were reported by 2 patients and included reversible sleep and mood problems, headache, and a sensation of heat.

CONCLUSIONS DBS telemedicine seems to offer a feasible, safe, and efficient strategy for maintaining the delivery of medical care to psychiatric patients during the COVID-19 outbreak. The authors propose that implementation of a comprehensive DBS telemedicine system, which combines DBS teleprogramming with psychological counseling, medical consultations, and medication prescriptions and delivery, could be an efficient and effective approach to manage the mental health and quality of life of patients with psychiatric disorders during future local or global public health crises.

https://thejns.org/doi/abs/10.3171/2020.9.FOCUS20666
\end{abstract}

KEYWORDS deep brain stimulation; case management; psychiatric disorder; COVID-19

$\mathrm{D}$ EEP brain stimulation (DBS) is a neurosurgical treatment option for severe and refractory cases of movement disorders and certain psychiatric disorders. Postoperative programming is a critical part of case management for patients who have undergone DBS surgery. However, the ongoing coronavirus disease 2019 (COVID-19) pandemic has exposed major weaknesses in the delivery of medical care to patients in whom DBS devices have been implanted. Specifically, many patients who had undergone DBS surgery were unable to attend initial routine postoperative DBS programming and optimization sessions due to the implementation of federal or local preventive regulations, such as social distancing, quarantines, and lockdowns. ${ }^{1}$ Recently, the guideline for DBS device management has been modified to take into account the COVID-19 pandemic, recommending that clinicians postpone initial and subsequent postoperative DBS programming and optimization sessions. ${ }^{1}$ However, such a

ABBREVIATIONS BAI = Beck Anxiety Inventory; BDI = Beck Depression Inventory; BNST = bilateral bed nucleus of the stria terminalis; DBS = deep brain stimulation; EDE-Q = Eating Disorder Examination Questionnaire; HAM-A = Hamilton Anxiety Rating Scale; HAM-D = Hamilton Depression Rating Scale; MDD = major depressive disorder; OCD = obsessive-compulsive disorder; SDS = Sheehan Disability Scale; SE-AN = severe and enduring anorexia nervosa; Y-BOCS = Yale-Brown ObsessiveCompulsive Scale.

SUBMITTED July 29, 2020. ACCEPTED September 18, 2020.

INCLUDE WHEN CITING DOI: 10.3171/2020.9.FOCUS20666. 
delay and interruption of routine medical care could compromise the mental and physical well-being of the patients involved, especially those with psychiatric disorders who are more vulnerable to social isolation. ${ }^{2}$

Fortunately, the use of novel DBS telemedicine programming technology can help maintain the delivery of routine medical care to patients during public health emergencies. ${ }^{3}$ Although DBS telemedicine technology is not yet widely available, in some countries, such as China, DBS telemedicine platforms were developed and clinically applied prior to the COVID-19 pandemic. ${ }^{4,5}$ These DBS telemedicine platforms, which incorporate synchronous real-time video communication, allow patients in remote locations to access specialized medical care rapidly and efficiently, without requiring them to travel. Similarly, DBS telemedicine permits clinicians to deliver DBS parameter adjustments as well as online psychological support, medical consultations, and prescriptions in a timely manner. Despite these advantages, the role and value of DBS telemedicine for psychiatric disorders in general and during public health emergencies specifically have not yet been well documented in the literature. Recently, we briefly described our initial experiences with DBS telemedicine in the treatment of 2 psychiatric patients during the COVID-19 pandemic. ${ }^{2}$ Here, we describe in greater detail 4 patients with psychiatric disorders who underwent DBS surgery at our institution and received multiple postoperative DBS telemedicine sessions during the COVID-19 pandemic.

\section{Methods \\ Participants}

We retrospectively reviewed the cases of 4 consecutive patients who underwent DBS surgery for severely disabling mental health problems at Ruijin Hospital between January 2020 and June 2020. Two patients suffered from severe obsessive-compulsive disorder (OCD), another patient from treatment-resistant major depressive disorder (MDD), and the remaining patient from severe and enduring anorexia nervosa (SE-AN). Each patient received regular postoperative DBS telemedicine case management during the COVID-19 pandemic. The patients had received their diagnoses from an experienced psychiatrist according to a standardized mental health examination based on the Diagnostic and Statistical Manual of Mental Disorders, Fifth Edition (DSM-5). All patients provided preoperative written informed consent to undergo surgery and to participate in follow-up examinations. Here, we report the clinical outcome of these patients after they received between 1 and 6 months of DBS treatment.

\section{DBS Device and Teleprogramming}

The targets of DBS were the bilateral bed nucleus of the stria terminalis (BNST) in the patients with OCD and the patient with SE-AN, and the bilateral habenula in the patient with MDD. Postoperative DBS programming was initiated approximately 3-4 weeks after electrode implantation to allow the microlesional effect of the surgery to resolve. ${ }^{6}$ The postoperative programming sessions, including the initial programming and subsequent parameter op- timizations, were all performed using DBS telemedicine technology to maintain the delivery of medical care when travel restrictions and other public health protective measures were put in place during the COVID-19 outbreak.

The characteristics of the intracranial nondirectional leads and the rechargeability of the neurostimulator were similar to those of DBS devices used in many other countries. The neurostimulator that we used had a special feature: it could be programmed wirelessly and remotely through a DBS telemedicine platform after the connection between the "external (doctor) programmer" and "patient client" components of the platform had been established. The DBS telemedicine platform that we used has been described previously. ${ }^{5}$ Briefly, the platform involved a synchronous system that allowed doctor-patient interactions via real-time encrypted video communications. The platform comprised four components: the doctor programmer, patient client, server station, and video communication system. Data transmission relied on either a home Wi-Fi or mobile network combined with a Bluetooth module. The initial DBS teleprogramming session was similar to conventional face-to-face DBS programming. During this session, the contacts were individually tested to explore the immediate DBS clinical effects as well as any stimulation-related side effects experienced by the patients. Generally, subsequent teleprogramming sessions for parameter optimization were planned every 2 weeks to take into account the more gradual and less predictable DBS effects in patients with psychiatric disorders, as compared with the DBS effects observed in patients with movement disorders. Additional online appointments could be scheduled at the patient's request. During each remote programming session, the different settings of DBS parameters used, notably the final setting that provided the clinically most favorable stimulation effects, were stored to enable the patient to switch between parameter settings or have the parameters adjusted by the patient programmer in case none worked well after the session.

\section{Results}

Table 1 presents for each patient the clinical outcome data that were obtained at the final follow-up assessment using the DBS telemedicine platform and well-established clinical symptom rating scales. One patient (case 1 ), who had been treated with BNST-DBS for OCD, displayed a modest improvement in social functioning (13\% score reduction on the Sheehan Disability Scale [SDS] ${ }^{7}$ at the 1-month follow-up examination relative to preoperative (baseline) functioning. Gradually, after 6 months of BNST-DBS treatment, this patient also exhibited a modest reduction in OCD symptom severity (15\% score reduction on the Yale-Brown Obsessive-Compulsive Scale [Y-BOCS] $),{ }^{8}$ along with marked reductions in comorbid symptoms of depression (61\% score reduction on the Beck Depression Inventory [BDI] $)^{9}$ and anxiety $(82 \%$ score reduction on the Beck Anxiety Inventory [BAI] $)^{10}$ (Table 1).

By comparison, the other patient with OCD (case 2), who also underwent BNST-DBS, demonstrated an exac- 


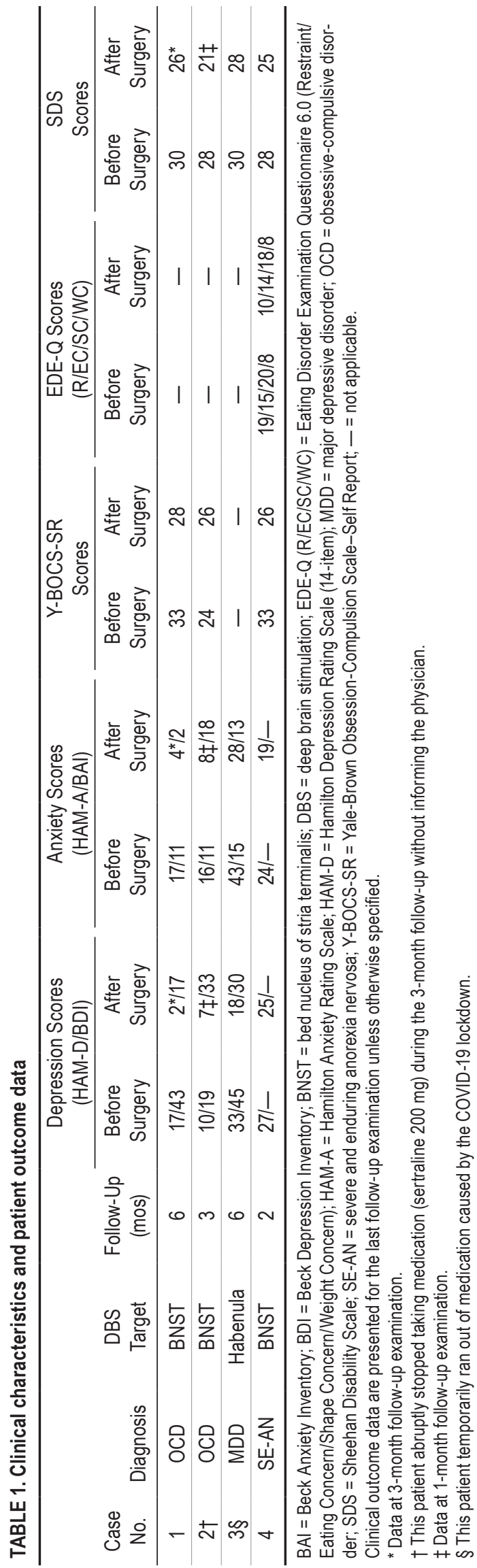

erbation of OCD symptoms ( $8 \%$ score increase on the YBOCS) and comorbid symptoms of depression (42\% score increase on the BDI) and anxiety (39\% score increase on the BAI) at the 3-month follow-up examination (Table 1), most likely because this patient had abruptly stopped taking the prescribed medications after surgery without seeking prior medical consultation. This case illustrates that DBS cannot yet serve as a substitute for pharmacotherapy and underlines the importance of patient compliance with continuing medications after DBS surgery.

The third patient (case 3), who underwent habenulaDBS for MDD, exhibited reductions in symptoms of both depression (33\% score decrease on the BDI and 46\% score decrease on the Hamilton Depression Rating Scale [HAM-D] $)^{11}$ and anxiety (13\% score decrease on the BAI and a 35\% score decrease on the Hamilton Anxiety Rating Scale [HAM-A] $)^{12}$ at the 6-month follow-up examination (Table 1). The clinical improvements observed after 6 months of habenula-DBS treatment were accompanied by a slight improvement in the patient's social functioning (7\% score decrease on the SDS). Of note, this patient ran out of medications following a disruption in the drug supply chain due to the COVID-19 outbreak, which was solved by a third-party online medication prescription and delivery platform.

The final patient (case 4), who underwent BNST-DBS for SE-AN, showed improvements in eating behavior, as assessed by the Eating Disorder Examination Questionnaire (EDE-Q) $)^{13}(47 \%$ score reduction on the Restraint subscale), as well as in comorbid symptoms of depression ( $7 \%$ score reduction on the HAM-D), anxiety ( $21 \%$ score reduction on the HAM-A), and OCD (21\% score reduction on the Y-BOCS) at the 2-month follow-up examination. The clinical improvements co-occurred with a slight improvement in the patient's social functioning (11\% score decrease on the SDS).

The 4 patients completed a total of 45 DBS telemedicine sessions during the COVID-19 outbreak (Table 2). Twenty-eight sessions (62\%) involved DBS teleprogramming. Adjustments were made to the DBS voltage level during 21 sessions, frequency in 12 sessions, pulse width in 9 sessions, and contact site in 9 sessions. Psychological support was provided to patients and some technical problems were solved during the other sessions. DBS-related side effects reported in case 1 included sleep and mood problems, and those reported in case 2 were headache and a sensation of heat. The side effects were resolved by parameter adjustments. No unexpected loss of network connection occurred during the sessions (Table 2).

\section{Discussion}

The results of this study add to the limited information currently available on the role and value of DBS telemedicine for delivering DBS treatment and care to patients with severe psychiatric disorders in general and during a global public health emergency specifically. To discuss the utility of DBS telemedicine management for psychiatric disorders, it may be informative to compare its use with DBS telemedicine case management in patients with movement disorders (e.g., Parkinson's disease 
TABLE 2. Number and nature of completed DBS teleprogramming sessions during the COVID-19 outbreak

\begin{tabular}{|c|c|c|c|c|c|c|c|}
\hline \multirow[b]{2}{*}{$\begin{array}{l}\text { Case } \\
\text { No. }\end{array}$} & \multirow[b]{2}{*}{$\begin{array}{l}\text { No. of DBS Telemedicine Sessions } \\
\text { (for programming/for other reasons) }\end{array}$} & \multicolumn{4}{|c|}{ Parameter Changes (no. of sessions) } & \multirow[b]{2}{*}{$\begin{array}{l}\text { Side Effects } \\
\text { Reported }\end{array}$} & \multirow{2}{*}{$\begin{array}{c}\text { Existence of } \\
\text { Disconnectior } \\
\text { Issues }\end{array}$} \\
\hline & & Voltage & Frequency & $\begin{array}{l}\text { Pulse } \\
\text { Width }\end{array}$ & $\begin{array}{l}\text { No. of Contact } \\
\text { Sites }\end{array}$ & & \\
\hline 1 & $12 / 7$ & 8 & 4 & 5 & 2 & $\begin{array}{l}\text { Sleep problem; } \\
\text { mood problem }\end{array}$ & No \\
\hline 2 & $3 / 5$ & 2 & 0 & 1 & 0 & $\begin{array}{l}\text { Reversible headache; } \\
\text { sensation of heat }\end{array}$ & No \\
\hline 3 & $11 / 5$ & 9 & 6 & 3 & 5 & - & No \\
\hline 4 & $2 / 0$ & 2 & 2 & 0 & 2 & - & No \\
\hline
\end{tabular}

or dystonia), which has been much better documented in the literature..$^{3,14}$ It seems that DBS telemedicine for psychiatric disorders is also feasible and safe and can yield significant clinical improvements in some cases, but this application may have several advantages relative to the use of DBS telemedicine to treat movement disorders. Initially, DBS telemedicine with an incorporated real-time video communication system seems to be more suitable for assessing a patient's mental health status than for examining the integrity of gross and fine motor functions. Indeed, telemedicine has already been applied successfully to a wide variety of medical conditions and can be used efficiently to treat a patient's mental health. ${ }^{15}$ In fact, several "telepsychiatry" guidelines have recently been published to ensure that the delivery of telemental healthcare to patients is safe and adequate. ${ }^{16,17}$ In contrast, the application of DBS telemedicine case management in patients with movement disorders has been challenging. The main reason for this is that a hands-on clinical assessment of a patient's motor functions is not possible, although it is required to provide ratings on the rigidity and retropulsion pull testing items of the Unified Parkinson's Disease Rating Scale (UPDRS), ${ }^{18}$ which is the most commonly used rating scale to assess motor function. Although a modified UPDRS for remote assessment has been proposed, ${ }^{19}$ its utilization has not been universally accepted. Another current disadvantage of DBS telemedicine management for movement disorders is related to internet connection issues, such as video transmission delay, which might considerably affect the rating of visually assessed motor items (e.g., finger tapping).

Furthermore, DBS is already an established and formally approved treatment option for movement disorders, but its application to the management of psychiatric disorders is still at an early stage of development and considered offlabel, and it remains under extensive investigation for these clinical conditions. Use of DBS in the management of psychiatric disorders will provide not only further impetus for the development of new, more sophisticated and effective DBS techniques, but also a better understanding of the brain structures and networks that mediate affective and cognitive dysfunctions basic to many psychiatric disorders. It is also reasonable to expect that DBS management of psychiatric disorders, which is currently more complicated and laborious than that for movement disorders, will become more efficient and affordable for patients when DBS telemedicine is used. For example, the DBS management of psychiatric disorders typically requires frequent followup face-to-face examinations for parameter optimization, which in turn may lead to an increase in the patient's travel and accommodation expenses. Thus, DBS telemedicine management of psychiatric disorders is anticipated to increase the efficiency, individual affordability, and access of patients to DBS treatment and care.

Several issues should be addressed to ensure the safety and reliability of DBS telemedicine management of psychiatric disorders during a pandemic. First, a stable internet connection provides the foundation for telemedicine management. Our clinical experience indicates that a wired or house Wi-Fi connection is more stable than a mobile network or mobile Bluetooth-based connection, because the latter connection can be disrupted by phone calls. Such an unexpected disconnection can result in undesirable stimulating effects, abrupt cessation of stimulation, or even malfunction of the neurostimulator. Second, various stored DBS parameter settings were required for DBS telemedicine management during the COVID-19 pandemic. This option is important because it would enable the patient to restore the previous stimulating parameter setting if symptoms deteriorated after the recent teleprogramming session, even in the scenario of limited access to conventional medical care services or the unavailability of DBS teleprogramming under certain rare circumstances. Finally, because one of our patients ran out of medication during the COVID-19 outbreak, we recommend the incorporation of an online prescription and medication delivery system into the DBS telemedicine management platform.

\section{Conclusions}

The COVID-19 pandemic has considerably changed and continues to change healthcare systems worldwide. DBS telemedicine seems to be an invaluable tool for the treatment of patients with psychiatric disorders during this public health emergency. The implementation of a comprehensive DBS telemedicine framework, which combines DBS teleprogramming with psychological counseling, medical consultation, and medication prescription and delivery, may be crucial to ensure the mental health and quality of life of psychiatric patients during future local or global public health crises. 


\section{Acknowledgments}

This study was partially supported by the Shanghai Clinical Research Center for Mental Health (19MC1911100) and SJTU Trans-med Awards Research (2019015).

We appreciate the patients' enduring trust during the unprecedented COVID-19 outbreak period.

\section{References}

1. Miocinovic S, Ostrem JL, Okun MS, et al. Recommendations for deep brain stimulation device management during a pandemic. J Parkinsons Dis. 2020;10(3):903-910.

2. Zhang C, Zhu K, Li D, et al. Deep brain stimulation telemedicine for psychiatric patients during the COVID-19 pandemic. Brain Stimul. 2020;13(5):1263-1264.

3. Zhang J, Hu W, Chen $\mathrm{H}$, et al. Implementation of a novel Bluetooth technology for remote deep brain stimulation programming: the pre- and post-COVID-19 Beijing experience. Mov Disord. 2020;35(6):909-910.

4. Chen Y, Hao H, Chen H, Li L. The study on a telemedicine interaction mode for deep brain stimulation postoperative follow-up. Annu Int Conf IEEE Eng Med Biol Soc. 2015; 2015:186-189.

5. Zhang C, Li D, Zeljic K, et al. A remote and wireless deep brain stimulation programming system. Neuromodulation. 2016;19(4):437-439.

6. Mann JM, Foote KD, Garvan CW, et al. Brain penetration effects of microelectrodes and DBS leads in STN or GPi. $J$ Neurol Neurosurg Psychiatry. 2009;80(7):794-797.

7. Sheehan DV, Harnett-Sheehan K, Raj BA. The measurement of disability. Int Clin Psychopharmacol. 1996;11(suppl 3): 389-395.

8. Storch EA, Rasmussen SA, Price LH, et al. Development and psychometric evaluation of the Yale-Brown ObsessiveCompulsive Scale-Second Edition. Psychol Assess. 2010; 22(2):223-232.

9. Beck AT, Steer RA, Ball R, Ranieri W. Comparison of Beck Depression Inventories-IA and -II in psychiatric outpatients. J Pers Assess. 1996;67(3):588-597.

10. Beck AT, Epstein N, Brown G, Steer RA. An inventory for measuring clinical anxiety: psychometric properties. J Consult Clin Psychol. 1988;56(6):893-897.

11. Hamilton M. A rating scale for depression. J Neurol Neurosurg Psychiatry. 1960;23(1):56-62.

12. Hamilton M. The assessment of anxiety states by rating. $\mathrm{Br} J$ Med Psychol. 1959;32(1):50-55.

13. Fairburn CG, Beglin SJ. Assessment of eating disorders: interview or self-report questionnaire? Int J Eat Disord. 1994; 16(4):363-370.

14. Jitkritsadakul O, Rajalingam R, Toenjes $\mathrm{C}$, et al. Tele-health for patients with deep brain stimulation: the experience of the Ontario Telemedicine Network. Mov Disord. 2018;33(3): 491-492.
15. Doarn CR. Telemedicine and psychiatry - a natural match. mHealth. 2018:4:60.

16. Shore JH, Yellowlees $\mathrm{P}$, Caudill R, et al. Best practices in videoconferencing-based telemental health April 2018. Telemed J E Health. 2018;24(11):827-832.

17. American Academy of Child and Adolescent Psychiatry (AACAP) Committee on Telepsychiatry and AACAP Committee on Quality Issues. Clinical update: telepsychiatry with children and adolescents. J Am Acad Child Adolesc Psychiatry. 2017;56(10):875-893.

18. Fahn S, Elton RL. Unified Parkinson's Disease Rating Scale. In: Fahn S, Marsden CD, Goldstein M, Calne DB, eds. Recent Developments in Parkinson's Disease. Macmillan Health Care, 1987:153-163.

19. Abdolahi A, Scoglio N, Killoran A, et al. Potential reliability and validity of a modified version of the Unified Parkinson's Disease Rating Scale that could be administered remotely. Parkinsonism Relat Disord. 2013;19(2):218-221.

\section{Disclosures}

The authors report no conflict of interest concerning the materials or methods used in this study or the findings specified in this paper.

\section{Author Contributions}

Conception and design: C Zhang. Acquisition of data: Y Zhang, Li. Analysis and interpretation of data: Lin. Drafting the article: Lin. Critically revising the article: C Zhang, Y Zhang, Dai, Li. Reviewed submitted version of manuscript: all authors. Approved the final version of the manuscript on behalf of all authors: $\mathrm{C}$ Zhang. Administrative/technical/material support: Sun. Study supervision: Voon, Sun.

\section{Correspondence}

Chencheng Zhang: Center for Functional Neurosurgery, Ruijin Hospital, Shanghai Jiao Tong University School of Medicine, Shanghai, China.i@cczhang.org. 